\title{
Uso de las TIC en el aprendizaje de las matemáticas en el nivel superior
}

\author{
Use of ICT in the Learning of Mathematics at the Higher Level \\ Uso das TIC na aprendizagem da matemática no nível superior
}

\author{
Angelino Feliciano Morales \\ Universidad Autónoma de Guerrero, México \\ afmorales@uagro.mx \\ https://orcid.org/0000-0002-7707-7319 \\ René Edmundo Cuevas Valencia \\ Universidad Autónoma de Guerrero, México \\ reneecuevas@uagro.mx \\ https://orcid.org/0000-0001-9528-7603
}

\section{Resumen}

Considerando que el aprendizaje de las matemáticas continúa siendo un problema nodal en México, este trabajo presenta una propuesta para utilizar las tecnologías de la información y comunicación (TIC) en el proceso de enseñanza-aprendizaje de las matemáticas en el nivel superior y así contribuir a la mejora del rendimiento académico de los educandos. La investigación se desarrolló en la Facultad de Ingeniería de la Universidad Autónoma de Guerrero (UAGro). Con un enfoque cuantitativo, de tipo exploratorio, se midió el impacto del uso de las TIC en la resolución de una ecuación diferencial no homogénea por medio del método de coeficientes indeterminados. Para ello, se evaluó tanto a un grupo control como a un grupo experimental y se realizó un cuestionario antes y después de la intervención. La importancia del uso de las herramienta tecnológicas se apreció en los resultados aquí conseguidos en el grupo experimental, donde 14 de 15 estudiantes aprobaron el nivel esperado. En consecuencia, el uso de las TIC favoreció el rendimiento académico de los estudiantes participantes del nivel superior. 


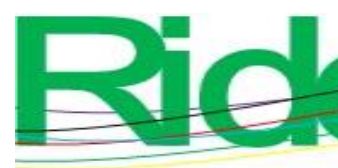

Revista Iberoamericana para la
Investigación y el Desarrollo Educativo
ISSN $2007-7467$

Palabras clave: aprendizaje formal, ecuación diferencial, enseñanza profesional, matemáticas, TIC.

\section{Abstract}

Considering that the learning of mathematics continues to be a nodal problem in Mexico, this work presents a proposal to use information and communication technologies (ICT) in the teaching-learning process of mathematics at the higher level and thus contribute to the improvement of the academic performance of students. The research was developed at the Faculty of Engineering of the Universidad Autónoma de Guerrero (UAGro). Using a quantitative, exploratory approach, the impact of the use of ICTs in solving a nonhomogeneous differential equation was measured. For this, both a control group and an experimental group were evaluated, and a questionnaire was carried out before and after the intervention. The importance of the use of technological tools was appreciated in the results obtained here in the experimental group, where 14 of 15 students passed the expected level. Consequently, the use of ICT favored the academic performance of the participating students of the higher level.

Keywords: formal learning, differential equation, professional teaching, mathematics, ICT.

\section{Resumo}

Considerando que a aprendizagem da matemática continua sendo um problema nodal no México, este trabalho apresenta uma proposta para utilizar as tecnologias de informação e comunicação (TIC) no processo de ensino-aprendizagem da matemática de nível superior e, assim, contribuir para melhorar o desempenho acadêmico dos alunos. A pesquisa foi desenvolvida na Faculdade de Engenharia da Universidade Autônoma de Guerrero (UAGro). Por meio de uma abordagem quantitativa e exploratória, o impacto do uso das TICs na solução de uma equação diferencial não homogênea foi medido por meio do método dos coeficientes indeterminados. Para isso, foram avaliados um grupo controle e um grupo experimental e realizado um questionário antes e após a intervenção. A importância da utilização de ferramentas tecnológicas foi apreciada nos resultados aqui obtidos no grupo experimental, onde 14 dos 15 alunos ultrapassaram o nível esperado. Consequentemente, o uso das TIC favoreceu o desempenho acadêmico dos alunos participantes do nível superior. 


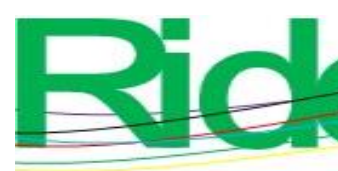

Revista Iberoamericana para la Investigación y el Desarrollo Educativo ISSN $2007-7467$

estancia universitaria, esté en posibilidades de competir en el mercado laboral y tenga un desempeño exitoso. Desde el punto de vista del docente, este debe esforzarse en estar actualizado en el uso pedagógico de las TIC en el proceso de enseñanza-aprendizaje de las matemáticas en el nivel superior, aquí de particular interés, o de cualquier otra área del saber y nivel educativo. En otras palabras, estar al tanto del avance de la tecnología, de modo que incida en el proceso de adquisición del conocimiento por parte del estudiantado y facilite la comprensión de los conceptos o operaciones entre los elementos u objetos de las matemáticas, en este caso.

Respecto a las características de esta investigación, adopta un enfoque cuantitativo para medir el impacto de las TIC en el rendimiento de los alumnos en la resolución de problemas matemáticos. Lo anterior a través de la implementación de una secuencia didáctica cuyo pilar es el uso de las TIC y con el propósito de crear un programa de tutorías, y de manera particular, una asesoría entre pares.

En el campo de las matemáticas existen investigaciones que justifican el uso de las TIC en la práctica docente. Dichos esfuerzos describen estrategias didácticas que han permitido la apropiación del conocimiento por parte de los alumnos de una manera fácil. Por supuesto, estos antecedentes fueron la base para la elaboración de una propuesta innovadora que fortaleciera la formación académica e intelectual de la comunidad que conforma la Facultad de Ingeniería de la UAGro.

\section{Antecedentes}

En el programa educativo de ingeniero en Computación de la Facultad de Ingeniería de la UAGro se ha observado un alto índice de reprobación en los primeros semestres de la carrera profesional, lo cual ha propiciado que en algunas ocasiones los alumnos abandonen sus estudios.

De acuerdo con Galán et al. (2009), las matemáticas desempeñan un triple papel en la ingeniería: instrumental, formativo y de fundamentación teórica. "En su papel instrumental, el conocimiento matemático proporciona técnicas y estrategias básicas para otras materias de estudio y para la actividad profesional” (p. 3). Respecto al papel formativo, “contribuyen a la mejora de estructuras mentales y a la adquisición de aptitudes cuya utilidad y alcance trascienden el ámbito de las propias matemáticas" (p. 3). Por último, en cuanto a la fundamentación teórica, "las definiciones, demostraciones y los encadenamientos 


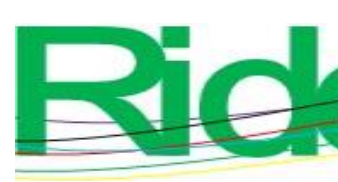

Revista Iberoamericana para la Investigación y el Desarrollo Educativo ISSN $2007-7467$

conceptuales y lógicos, en tanto que dan validez a las intuiciones y confieren sentido a las técnicas aplicadas, deben ser introducidos en estos estudios con un cierto nivel formal" (p. $3)$.

Ahora bien, Romo, Lemarie y Vidal (2013, p. 222) realizaron un trabajo sobre las condiciones pedagógicas de Internet y de diferentes herramientas de la web 2.0 en el nivel de secundaria, que contempla el capítulo de la ecuación de la recta. En el diseño metodológico, fueron considerados dos grupos, uno experimental y otro de control. Con el grupo experimental, se abordaron los contenidos de la unidad de matemáticas utilizando herramientas web 2.0. En el grupo de control, se trabajó con guías de aprendizaje y medios tradicionales. En ambos grupos se administró una posevaluación. En los resultados se encontró que los estudiantes que realizaron actividades pedagógicas innovadoras obtuvieron mejores resultados que aquellos del grupo de control, que trabajaron con guías de aprendizaje de forma tradicional.

En esa misma línea, Sánchez (2012, p. 27) establece que los recursos tecnológicos deben tener una aplicación pedagógica y apoyarse en las diferentes teorías propuestas por didactas de las matemáticas; Sánchez (2012) enfatiza en la dimensión semiótica de este lenguaje. La didáctica de las matemáticas, como ciencia pedagógica, centra su estudio en los procesos y fenómenos resultantes ocurridos dentro del aula y fuera de ella.

Desde la perspectiva socializadora de esta teoría, las TIC permiten tender puentes al ofrecer herramientas comunicativas y operacionales con la finalidad de concretar la construcción del conocimiento. Los sistemas educativos abiertos, por su parte, dependerán de las condiciones ofrecidas por los centros educacionales y las características particulares de los estudiantes que propicien el intercambio comunicacional con otros grupos formados para el aprendizaje de las matemáticas.

Avilés, Feliciano, Cuevas y Alonso (2015, p. 10) lograron evidenciar la importancia que tiene la aplicación del software matemático en línea GeoGebra para determinar máximos y mínimos de una función de variable real. La utilización de esta herramienta ha permitido al estudiante desarrollar habilidades de cálculo diferencial y confirmar la eficiencia del software. Estos aprendizajes dieron certeza al estudiante sobre la importancia que tiene el uso del software en la docencia, lo cual facilitó el logro de la competencia de la unidad de aprendizaje. Asimismo, Feliciano, Cuevas y Catalán (2016, p. 84) corroboraron la eficacia de GeoGebra como herramienta auxiliar docente en el cálculo del área entre dos funciones reales. 


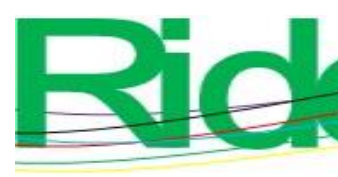

\section{Revista Iberoamericana para la Investigación y el Desarrollo Educativo ISSN 2007 - 7467}

Otros ejemplos de estas herramientas tecnológicas con fines educativos son los calculadores de matrices, por ejemplo, Matrix calculator y Resolver Matrices. Ambos softwares resuelven problemas elementales de matrices y se encuentran disponibles en http://www.resolvermatrices.com/ y https://matrixcalc.org/es/, respectivamente, donde se ofrece al usuario la oportunidad de realizar las operaciones indicadas (Nieto, Cuevas y Feliciano, 2016).

Por otra parte, Feliciano y Cuevas (2017, p. 53) describieron el proceso para elaborar videotutoriales con el software Windows Movie Maker. A manera de ejemplo, los autores crearon un videotutorial que puede ser utilizado por docentes y estudiantes del nivel superior que estén interesados en el aprendizaje del cálculo integral. Así, demostraron a los profesores que es posible preparar material didáctico visual que facilite la presentación del contenido de las matemáticas.

El avance de la tecnología en el desarrollo de dispositivos móviles también ha sido impresionante. Atrás quedaron aquellos aparatos que solo servían para llamar y enviar mensajes. En el ámbito educativo, uno de los primeros cambios de este proceso evolutivo fue que el móvil prácticamente sustituyera a la calculadora, debido a la facilidad de encontrar un software matemático en la Internet e instalarlo en el celular para convertirlo en una herramienta de apoyo para la enseñanza-aprendizaje de las matemáticas. Ahora programas como GeoGebra, MathPapa y Mathway son bastante populares para calcular, por ejemplo, raíces de un polinomio de grado mayor a tres (Maximino, Cuevas, Guinto y Feliciano, 2017).

Por supuesto, la línea evolutiva de estas herramientas digitales aplicadas dentro del aula escolar ha sido acompañada de referentes teóricos y pedagógicos como la teoría conductista, la cognitiva, la constructivista y la reciente teoría sociocultural (Fernández, 2017). Estas teorías han evidenciado las transformaciones que se han dado en materia educativa a partir de la incorporación de las tecnologías digitales y el uso del computador. Sin embargo, la matemática ha sido uno de los campos del saber que más ha tardado en incorporar estas estrategias hacia la utilización de las TIC como apoyo en los procesos de aprendizaje. En el aprendizaje de las matemáticas el uso de currículos estructurados y secuenciales ha sido la base para adquirir habilidades procedimentales, esenciales en el abordaje de conceptos matemáticos (Vega, Niño y Cárdenas, 2015). 


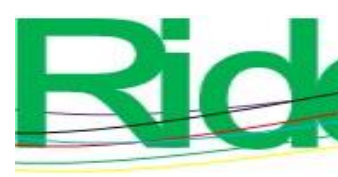

Revista Iberoamericana para la
Investigación y el Desarrollo Educativo
ISSN $2007-7467$

Al respecto, Triana, Ceballos y Villa (2016) recuperan, entre los testimonios de los participantes de su estudio, la importancia de que se desarrollen habilidades de reflexión y discusión en torno a los temas matemáticos e ir más allá de lo memorístico y mecánico. Esto establece un punto de partida esencial para el desarrollo de recursos interactivos como apoyo a la enseñanza y el aprendizaje de la matemática. Un caso particular que evidencia la trascendencia del uso de herramientas tecnológicas en procesos de educación matemática se encuentra en Ramírez (2015, citado en Grisales, 2018), quien, a través del software Mathematica 10, desarrolló una serie de herramientas que facilitan el aprendizaje de ciertos temas de precálculo con estudiantes universitarios.

Y volviendo a los referentes pedagógicos, David Ausubel, un psicólogo educativo de los años 60, dejó un importante legado a través de sus elaboraciones teóricas sobre la forma en que se realiza la actividad intelectual en el ámbito escolar. Su obra y la de algunos seguidores han marcado las deficiencias de la psicología de la educación, en especial sobre el movimiento cognoscitivista. La postura de Ausubel se caracteriza como constructivista e interaccionista; para él, los materiales de estudio y la información exterior se interrelacionan e interactúan con los esquemas de conocimiento previo y las características personales del aprendiz (Díaz, 1989, citada en Díaz y Hernández, 2015). Asimismo, concibe al estudiante como un procesador activo de la información, y afirma que el aprendizaje es sistemático y organizado, dado que es un fenómeno complejo. A pesar de lo importante que es el aprendizaje por descubrimiento, se considera que no es factible que todo el aprendizaje significativo que ocurre en el aula debe ser por esta opción. Sin embargo, impulsa el aprendizaje verbal significativo, el cual permite el dominio de contenidos curriculares que se imparten en las escuelas, sobre todo a nivel medio y superior.

Por último, Díaz y Hernández (2015) mencionan que el aprendizaje implica una reestructuración activa de las percepciones, ideas, conceptos y esquemas que el aprendiz posee en su estructura cognitiva. 


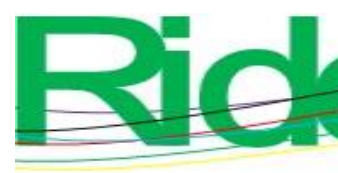

Revista Iberoamericana para la Investigación y el Desarrollo Educativo ISSN $2007-7467$

\section{Metodología}

Siguiendo a Hernández y Mendoza (2018, p. 106), esta investigación se ubica en el campo exploratorio, debido a que no existen documentos escritos sobre el diseño de estrategias didácticas utilizando las TIC en el proceso del aprendizaje de la resolución de una ecuación diferencial no homogénea por el método de coeficientes indeterminados en el nivel superior. No obstante, es importante señalar la existencia de algunos materiales aislados: libros, software matemático y videos. Por lo antes expuesto, se analizaron algunas herramientas para agruparlas según su funcionalidad y utilizarlas de manera combinada en la enseñanza de las matemáticas en el nivel superior.

Para obtener información se consideraron dos grupos: uno experimental y otro de control. Para el primero, se diseñó una secuencia didáctica utilizando las TIC. Mientras que en el grupo de control, se trabajó de manera tradicional, sin utilizar estrategias didácticas complementarias. Para ambos grupos se diseñaron dos cuestionarios: uno para antes de la intervención y otro para después. Cabe señalar que estos cuestionarios fueron idénticos para ambos grupos pero diferentes para cada momento y que fueron avalados por seis expertos, dos de ellos con un perfil de docencia, tres con perfil de ingeniería y uno con perfil del área de las matemáticas (Campbell y Stanley, 1970, p. 11).

La hipótesis planteada en el trabajo de investigación se basó en la supuesta valoración del impacto del uso de estrategias didácticas TIC bajo un enfoque constructivista en el aprendizaje de las matemáticas. Se trata de un esfuerzo para mejorar el rendimiento académico del estudiante del programa de ingeniero en Computación de la UAGro.

\section{Resultados}

Se aplicaron dos cuestionarios, en dos etapas, a los grupos de control y experimental: 15 preguntas cerradas de opción múltiple basadas en la resolución de una ecuación diferencial no homogénea por medio del método de coeficientes indeterminados. El primero como pretest y el segundo como postest, con el mismo tipo de preguntas, pero sin ser iguales. El grupo de control estuvo integrado por 15 estudiantes de la generación 2017 del programa de licenciatura de Ing. en Computación (5..$^{\circ}$ semestre, turno matutino). Mientras que el grupo experimental estuvo conformado por 15 estudiantes de nuevo ingreso de la maestría en Ingeniería para la Innovación y Desarrollo Tecnológico. 


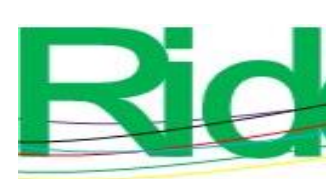

Revista Iberoamericana para la Investigación y el Desarrollo Educativo ISSN $2007-7467$

\section{Resultados de la prueba pretest}

En la tabla 1 se muestran los resultados obtenidos del pretest aplicado a los grupos de control y experimental.

Tabla 1. Resultados de la prueba pretest

\begin{tabular}{|c|c|c|c|}
\hline \multicolumn{2}{|l|}{ Grupo de control } & \multicolumn{2}{|l|}{ Grupo experimental } \\
\hline Estudiantes & $\begin{array}{l}\text { Número de } \\
\text { reactivos } \\
\text { correctos }\end{array}$ & Estudiantes & $\begin{array}{l}\text { Número de } \\
\text { reactivos } \\
\text { correctos }\end{array}$ \\
\hline $\mathrm{E}_{2}$ & 1 & $\mathrm{E}_{1}, \mathrm{E}_{2}$ & 1 \\
\hline $\mathrm{E}_{10}$ & 2 & $\mathrm{E}_{4}, \mathrm{E}_{5}, \mathrm{E}_{6}, \mathrm{E}_{10}$ & 2 \\
\hline $\begin{array}{l}E_{6}, E_{7}, E_{9}, E_{12}, \\
E_{14}\end{array}$ & 3 & $\mathrm{E}_{7}, \mathrm{E}_{9}, \mathrm{E}_{11}$ & 3 \\
\hline $\mathrm{E}_{3}, \mathrm{E}_{4}, \mathrm{E}_{5}, \mathrm{E}_{8}$ & 4 & $\begin{array}{l}E_{3}, E_{12}, E_{13}, E_{14}, \\
E_{15}\end{array}$ & 4 \\
\hline $\mathrm{E}_{1}, \mathrm{E}_{11}$ & 5 & $\mathrm{E}_{8}$ & 5 \\
\hline
\end{tabular}

Fuente: Elaboración propia

Se observa que se obtuvo un desempeño similar en ambos grupos, lo cual no es tan relevante, dado que estos resultados corresponden a la aplicación del instrumento para recabar la información antes iniciar las actividades del tema, que se refiere a la resolución de una ecuación diferencial no homogénea por el método de coeficientes indeterminados.

\section{Resultados de la prueba postest}

En la tabla 2 se encuentran los resultados obtenidos de la aplicación del postest a los dos grupos participantes en la investigación. Cabe recordar que tanto el de control como el experimental tuvieron un total de 15 integrantes. 
Tabla 2. Resultados de la prueba postest

\begin{tabular}{|l|c|l|c|}
\hline \multicolumn{2}{|l|}{ Grupo de control } & \multicolumn{2}{l|}{ Grupo experimental } \\
\hline Estudiantes & $\begin{array}{l}\text { Número de reactivos } \\
\text { correctos }\end{array}$ & Estudiantes & $\begin{array}{l}\text { Número de reactivos } \\
\text { correctos }\end{array}$ \\
\hline $\mathrm{E}_{5}$ & 6 & - & - \\
\hline $\mathrm{E}_{6,}, \mathrm{E}_{10}, \mathrm{E}_{12}$ & 7 & $\mathrm{E}_{10}$ & 7 \\
\hline $\mathrm{E}_{1}, \mathrm{E}_{2}, \mathrm{E}_{4}$ & 8 & $\mathrm{E}_{2}, \mathrm{E}_{3}, \mathrm{E}_{5}, \mathrm{E}_{9}$ & 8 \\
\hline $\mathrm{E}_{7}, \mathrm{E}_{11}, \mathrm{E}_{14}$ & 9 & $\mathrm{E}_{6}, \mathrm{E}_{7}$ & 10 \\
\hline $\mathrm{E}_{9} \mathrm{E}_{13}$ & 10 & $\mathrm{E}_{8}$ & 12 \\
\hline $\mathrm{E}_{3} \mathrm{E}_{15}$, & 11 & $\mathrm{E}_{11} \mathrm{E}_{12} \mathrm{E}_{14} \mathrm{E}_{15}$ & 13 \\
\hline $\mathrm{E}_{8}$ & 12 & $\mathrm{E}_{4}$ & 12 \\
\hline \multicolumn{1}{|c|}{} & - & $\mathrm{E}_{1}, \mathrm{E}_{13}$, & \\
\hline
\end{tabular}

Fuente: Elaboración propia (Feliciano, 2020)

También es importante señalar que se consideró un mínimo de ocho respuestas correctas, por lo que se puede afirmar que 11 integrantes del primer grupo lograron la competencia esperada, mientras que 14 individuos del segundo alcanzaron la competencia deseada.

\section{Comparación de resultados}

En primer lugar, se observó que 11 estudiantes del grupo de control consiguieron la meta propuesta, lo que representa $73 \%$ de aprovechamiento, mientras que 14 integrantes del experimental lograron el objetivo, lo que representa $93 \%$. Esto significa que el uso de las herramientas TIC impactó positivamente en la resolución de una ecuación diferencial no homogénea por medio del método de coeficientes indeterminados. Por lo anterior, se puede decir que el uso de las TIC favoreció el aprendizaje de las matemáticas en el nivel superior y de manera particular en el programa de ingeniero en Computación de la UAGro, lo cual, sobre todo, permite sugerir el uso de la herramienta tecnológica como apoyo didáctico en el aprendizaje de las matemáticas para el nivel superior. 


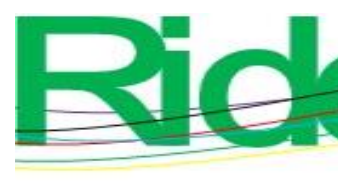

Revista Iberoamericana para la
Investigación y el Desarrollo Educativo
ISSN $2007-7467$

\section{Discusión}

La tecnología educativa, según Cabero (2007) y Belloch (2012), pasó de ser una herramienta estática a una viva, dinámica. En efecto, ahora es un apoyo didáctico para la enseñanza que favorece el desarrollo de habilidades en el estudiante para aplicarlas en su entorno. Cabero (2007), además, señala que la tecnología educativa es una de las disciplinas que más ha evolucionado y que, no está de más definirla, estudia los procesos de enseñanza y de transmisión de la cultura mediados tecnológicamente en distintos contextos educativos.

Aunado a lo anterior, hay que traer a la mesa los cambios internos y externos de las TIC en su conceptualización y aplicación: ya no se conciben como herramientas transmisoras de información, sino como instrumentos de pensamiento y cultura. Desde esta perspectiva, se justifica que las TIC sean elementos didácticos, educativos y herramientas intelectuales. Históricamente se registraron tres momentos evolutivos: 1) la inserción de los medios, 2) la concepción de la tecnología educativa por la aplicación de la psicología conductista y 3) el sustento de la teoría de sistemas y el enfoque sistémico aplicado a la educación (Cabero, 2003, 2007).

Ahora bien, Belloch $(2011,2012)$ afirma que la tecnología educativa se ubica en el ámbito de la didáctica y su desarrollo se ha dado a partir de los avances científicos producidos en los ámbitos de la informática y las telecomunicaciones. Los cambios que produce el uso de las TIC en el ámbito educativo han logrado que la atención no se centre tanto en el profesor y la enseñanza y más en el estudiante y el proceso de aprendizaje y en construir alternativas con los postulados del estudio socioconstructivo y los principios de la educación significativa (Belloch, 2011, 2012).

Respecto a los autores principales abordados en este trabajo, quienes coinciden en trabajar contenidos de ecuaciones diferenciales incorporando las TIC, afirman la importancia del uso de software para desarrollar esta actividad, aplicando estrategias didácticas e interactuando con los estudiantes. Como podrá observarse, la propuesta presentada de elaborar estrategias didácticas utilizando una herramienta tecnológica para abordar el contenido de la resolución de una ecuación diferencial no homogénea por medio del método de los coeficientes indeterminados es solo una manera más de incorporar las TIC en el proceso enseñanza-aprendizaje de las matemáticas. 


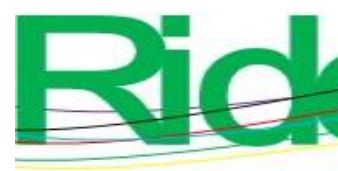

Revista Iberoamericana para la
Investigación y el Desarrollo Educativo
ISSN $2007-7467$

La importancia del uso de la herramienta tecnológica se aprecia en los resultados obtenidos del grupo experimental, donde 14 de 15 estudiantes alcanzaron el nivel esperado. Cabe resaltar que se consideraron como mínimo aceptable ocho respuestas correctas. Esto significa que el uso de la tecnología favoreció en la resolución de una ecuación diferencial no homogénea a través del método de coeficientes indeterminados.

Por lo anterior, se infiere que el uso de las TIC impactó favorablemente en el aprendizaje de las matemáticas en el nivel superior y de manera particular en el programa de ingeniero en Computación de la UAGro. Así pues, se recomienda utilizar la herramienta tecnología en el aprendizaje de las matemáticas en la UAGro.

\section{Conclusiones}

Una vez recorrido el camino hasta este punto se está en posibilidades de responder las interrogantes planteadas al inicio del presente trabajo de investigación.

Si la metodología utilizada en la estrategia didáctica facilita el aprendizaje de las matemáticas, el estudiante estará motivado con la incorporación de las TIC en su formación académica. Además, dado que el escolar está motivado con el uso de la herramienta tecnología en su adiestramiento matemático, puede construir un aprendizaje significativo.

Un aprendizaje es significativo para el estudiante si este ha sido adquirido a partir de los conocimientos previos. En efecto, si este ha sido adquirido a partir de los conocimientos precursores, ha generado un conocimiento relacional sobre la estructura conceptual del estudiante.

El uso de la herramienta tecnológica permitió que el estudiante se haya centrado en el planteamiento más que en el trabajo rutinario y que resolviera con cierta facilidad una ecuación diferencial no homogénea por medio del método de coeficientes indeterminados. Pero sobre todo, la estrategia didáctica implementada en la propuesta favoreció el uso de la herramienta tecnológica en la resolución de problemas. Efectivamente, la valoración de la estrategia didáctica por parte de los educandos ha sido buena, es decir, existe un grado elevado de satisfacción por parte de los escolares respecto al uso de la TIC. 


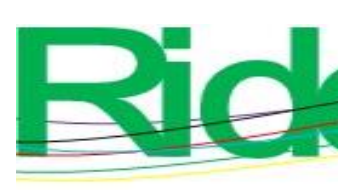
Revista Iberoamericana para la
Investigación y el Desarrollo Educativo
ISSN $2007-7467$

\section{Futuras Líneas de Investigación}

Pese a ser un gran avance, la información obtenida en esta investigación se considera insuficiente, por lo que es pertinente realizar nuevas exploraciones para responder a las inquietudes de los estudiantes de la Facultad de Ingeniería de la UAGro. En primer lugar, se debe impulsar la incorporación de las TIC para abordar los contenidos de la asignatura Ecuaciones Diferenciales, así como en las demás unidades de aprendizaje del área de las matemáticas de las carreras universitarias. En segundo, bajo esta orientación, se tiene en mente un próximo trabajo sobre sólidos de revolución correspondiente a la asignatura de Cálculo Integral, incluida en los planes de estudio de las cuatro carreras que se ofertan en la Facultad de Ingeniería, utilizando herramientas computacionales acordes a los tiempos; en particular, se pretende utilizar GeoGebra, que está siendo de gran ayuda en los diferentes niveles educativos, desde un enfoque metodológico y un formato multimodal.

\section{Referencias}

Avilés, J. E., Feliciano, A., Cuevas, R. E. y Alonso, A. G. (2015). Aplicación de GeoGebra en la determinación de máximos y mínimos en línea. Ponencia presentada en el 5. Congreso Internacional de Computación México-Colombia. Cartagena, del 24 al 26 de septiembre de 2015.

Belloch, C. (2011). Las tecnologías de la información y comunicación (TIC). (Material docente). Universidad de Valencia, Valencia.

Belloch, C. (2012). Las tecnologías de la información y comunicación en el aprendizaje. (Material docente). Universidad de Valencia, Valencia.

Cabero, J. (2003). Replanteando la tecnología educativa. Comunicar, (21), 23-30.

Cabero, J. C. (2007). Tecnología educativa: su evolución histórica y su conceptualización. En Cabero, J. C. (coord.), Tecnología educativa (pp. 13-28). México: McGraw-Hill.

Campbell, D. y Stanley, J. (1970). Diseños experimentales y cuasiexperimentales en la investigación social. Buenos Aires, Argentina: Amorrortu ediciones.

Díaz, A. y Hernández, R. (2015). Estrategias docentes para un aprendizaje significativo: una interpretación constructivista (2. ${ }^{\mathrm{a}}$ ed.). México: McGraw-Hill.

Feliciano, A. y Cuevas, R. E. (2017). Elaboración de videotutoriales con Windows Movie Maker para el aprendizaje de la matemática. Cómputo Aplicado, 1(2), 53-62. 


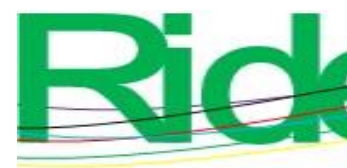

Revista Iberoamericana para la
Investigación y el Desarrollo Educativo
ISSN $2007-7467$

Feliciano, A., Cuevas, R. E. y Catalán, A. (2016). Aplicación de GeoGebra para calcular el área entre dos funciones. Tlamati, 7(3), 84-90.

Fernández, S. (2017). Evaluación y aprendizaje. marcoELE: Revista de Didáctica Español Lengua Extranjera, (24), 3.

Galán, J. L., Galán, M. A., Gálvez, A., Jiménez, A. J., Padilla, Y. y Rodríguez, P. (2009). Desde la calculadora hasta el software especializado: un estudio de la evolución en el uso de las TIC en la enseñanza de las matemáticas en ingeniería. Ponencia presentada en el XII Congreso de Informática en la Educación. Recuperado de http://www.bvs.hn/cu-2007/ponencias/EDU/EDU109.pdf.

Grisales, A. M. (2018). Uso de recursos TIC en la enseñanza de las matemáticas: retos y perspectivas. Entramado, 14(2), 198-214.

Hernández, R. y Mendoza, C. P. (2018). Metodología de la investigación. Las rutas cuantitativa, cualitativa y mixta. Ciudad de México, México: McGraw-Hill.

Maximino, G., Cuevas, R. E., Guinto, E. R. y Feliciano, A. (2017). Videotutoriales para el aprendizaje de la matemática usando dispositivos móviles. (Tesis de licenciatura) Universidad Autónoma de Guerrero, Chilpancingo.

Nieto, L. N., Valencia, R. E. Y Felicianos, A. (2016). Herramienta de ingeniería para el estudiante: calculador de matrices. Vínculos, 13(1), 56-64.

Romo, C., Lemarie, F. y Vidal, L. (2013). Aplicación del uso de Tecnologías educativas en la enseñanza de la matemática. Vínculos, 10(1), 221-226.

Ramírez, C. A. (2015). Diseño de herramientas que fomentan el aprendizaje de matemáticas con ayuda de Mathematica 10. Elementos, 5(5).

Sánchez, A. (2012). Incorporación de las TIC en el aprendizaje de la matemática en el sector universitario. Educación Matemática, 27(3).

Triana, M. M., Ceballos, J. F. y Villa, J. A. (2016). Una dimensión didáctica y conceptual de un instrumento para la valoración de objetos virtuales de aprendizaje. El caso de las fracciones. Entramado, 12(2), 166-186.

Vega, J. C., Niño, F. y Cárdenas, Y. P. (2015). Enseñanza de las matemáticas básicas en un entorno e-Learning: un estudio de caso de la Universidad Manuela Beltrán Virtual. Revista EAN, (79), 172-187. 


\begin{tabular}{|c|c|}
\hline$A$ & $\begin{array}{l}\text { Revista Iberoamericana para la } \\
\text { Investigación y el Desarrollo Educativo } \\
\text { ISSN } 2007-7467\end{array}$ \\
\hline Rol de Contribución & Autor (es) \\
\hline Conceptualización & Angelino Feliciano Morales \\
\hline Metodología & Angelino Feliciano Morales \\
\hline Software & $\begin{array}{l}\text { Angelino Feliciano Morales } \\
\text { Rene Edmundo Cuevas Valencia <<igual >> }\end{array}$ \\
\hline Validación & Rene Edmundo Cuevas Valencia \\
\hline Análisis Formal & Angelino Feliciano Morales \\
\hline Investigación & $\begin{array}{l}\text { Angelino Feliciano Morales } \\
\text { Rene Edmundo Cuevas Valencia «que apoya» }\end{array}$ \\
\hline Recursos & Angelino Feliciano Morales \\
\hline Curación de datos & $\begin{array}{l}\text { Angelino Feliciano Morales } \\
\text { Rene Edmundo Cuevas Valencia «que apoya» }\end{array}$ \\
\hline $\begin{array}{l}\text { Escritura - Preparación del } \\
\text { borrador original }\end{array}$ & $\begin{array}{l}\text { Angelino Feliciano Morales } \\
\text { Rene Edmundo Cuevas Valencia «que apoya» }\end{array}$ \\
\hline $\begin{array}{l}\text { Escritura - Revisión y } \\
\text { edición }\end{array}$ & $\begin{array}{l}\text { Angelino Feliciano Morales } \\
\text { Rene Edmundo Cuevas Valencia «que apoya» }\end{array}$ \\
\hline Visualización & $\begin{array}{l}\text { Angelino Feliciano Morales } \\
\text { Rene Edmundo Cuevas Valencia <<igual }>>\end{array}$ \\
\hline Supervisión & $\begin{array}{l}\text { Angelino Feliciano Morales } \\
\text { Rene Edmundo Cuevas Valencia «que apoya» }\end{array}$ \\
\hline Administración de Proyectos & Angelino Feliciano Morales \\
\hline Adquisición de fondos & Angelino Feliciano Morales \\
\hline
\end{tabular}

